\title{
A STUDY OF THE PROXIMAL RELATION IN COSET TRANSFORMATION GROUPS
}

\author{
BY \\ HARVEY B. KEYNES( $\left.{ }^{1}\right)$
}

1. Introduction. In this paper, we investigate the proximal relation and related notions in coset transformation groups. In $\S 1$, we show that many of the interesting properties of the proximal relation can be characterized in terms of a group subset that arises naturally from the generating subgroup. A general criterion for distal in terms of subnormality of the generating subgroup is shown in $\$ 2$. Proofs are given for [9, Remark (2.1) and Theorem (2.2)], establishing that coset transformation groups of nilpotent groups are distal. $\S 3$ is concerned with distal in relation to certain coset transformation groups that arise from solvable Lie groups. Finally, we establish in $\$ 4$ some results concerning the existence of subgroups which yield either point-transitive or minimal distal actions on the phase space. These results are strengthened when applied to certain discrete flows and examined in Lie groups. Proofs for the rest of the results of [9] are given in this section.

Let $G$ be a topological group and $H$ a subgroup of $G$. Consider the space $H \backslash G=\{H g \mid g \in G\}$ of right cosets of $G$. The coset transformation group of $G$ induced by $H$ is defined to be the point-to-point transitive transformation group $(H \backslash G, G)$ with action $(H f, g) \rightarrow H f g$. The fact that $(H \backslash G, G)$ is indeed a transformation group under this action follows from [6, Definition 1.39]. If $H$ is syndetic in $G$, $H \backslash G$ is compact uniformizable, whence $H \backslash G$ has a unique compatible uniformity. Thus, the proximal relation of $(H \backslash G, G)$ is well defined and will be denoted by $P(H \backslash G)$.

Throughout this paper, $G$ will denote a topological group with identity $e$ and $H$ will denote a syndetic subgroup. If $K$ and $L$ are subgroups of $G$, we denote that $K$ is normal in $L$ by $K \triangleleft L$. If $K$ is a subgroup of $G$, then $K^{*}$ will denote the closure of $K$. The additive groups of integers and reals will be denoted by $\boldsymbol{Z}$ and $\boldsymbol{R}$ respectively. The neighborhood filter of $e$ will be denoted by $\eta_{e}$. For other definitions, see [9].

Finally, the author would like to thank Professor W. Gottschalk for his stimulating comments concerning the results in this paper.

2. The proximal relation. When the phase space is not necessarily uniform, we can still introduce a notion of proximal.

Received by the editors October 31, 1966.

(1) These results form a portion of a doctoral dissertation written at Wesleyan University while the author held a National Science Foundation Cooperative Graduate Fellowship. 
Definition (2.1). Let $(X, T)$ be a transformation group, and $x, y \in X$. Then $x$ and $y$ are topologically proximal if there exists $z \in X$ such that for every neighborhood $U$ of $z$, there exists $t \in T$ such that $\{x t, y t\} \subset U$. $x$ and $y$ are topologically distal when they are not topologically proximal. $(X, T)$ is topologically distal if every pair of distinct points is topologically distal.

When the phase space is compact uniform, the two notions of proximal coincide.

REMARK. (2.2) Let $(X, T)$ be a transformation group with a compact uniform phase space. Let $x, y \in X$. Then the following statements are equivalent:

(1) $x$ and $y$ are topologically proximal.

(2) $x$ and $y$ are (uniformly) proximal.

Proof. We show that $x$ and $y$ are topologically distal iff $x$ and $y$ are distal. Suppose $x$ and $y$ are distal, Let $\alpha$ be an index of $X$ such that $(x, y) T \cap \alpha=\varnothing$. Let $\beta$ be a symmetric index such that $\beta^{2} \subset \alpha$. If $z \in X$ and $t \in T$ such that $\{x t, y t\} \subset z \beta$, then $(x t, y t) \subset z \beta \times z \beta \subset \beta^{2} \subset \alpha$, which is a contradiction. Then $x$ and $y$ are topologically distal.

Suppose $x$ and $y$ are topologically distal. Then every $z \in X$ has a neighborhood $U$ such that for all $t \in T,\{x t, y t\} \notin U$. Let $\alpha_{z}$ be an index such that $z \alpha_{z}^{2} \subset U$. Now $X=\bigcup_{z \in F} z \alpha_{z}$ for some finite subset $F$. Consider the index $\alpha=\bigcap_{z \in F} \alpha_{z}$. Suppose that for some $t \in T,(x t, y t) \in \alpha$. If $z \in F$ such that $x t \in z \alpha_{z}$, then $y t \in x t \alpha \subset x t \alpha_{z} \subset z \alpha_{z}^{2}$, and $\{x t, y t\} \subset z \alpha_{z}^{2} \subset U$. From this contradiction, it follows that $x$ and $y$ are distal.

The proof of (2.2) shows that when $X$ is simply uniform, if $x$ and $y$ are topologically proximal, then they are proximal.

We now show that $P(H \backslash G)$ depends on a certain subset of $G$.

THEOREM (2.3). Let $f, g \in G$. Then the following statements are pairwise equivalent:

(1) $(H f, H g) \in P(H \backslash G)$.

(2) $g f^{-1} \in \bigcap_{U \in n_{e}} H U H$.

(3) $e \in \operatorname{cl}\left(H g f^{-1} H\right)$.

Proof. We show that (1) implies (2). By (2.3), we can use the notion of topologically proximal. If $(H f, H g) \in P(H \backslash G)$, then there exists $d \in G$ such that for all $U \in \eta_{e}$, there exists $k \in G$ such that $\{H f k, H g k\} \subset H U d$. Coming back to $G$, we have that $H f k \cup H g k \subset H U d$. It then follows that $k \in f^{-1} H U d \cap g^{-1} H U d$, whence $f^{-1} H U d \cap g^{-1} H U d \neq \varnothing$, and $f^{-1} H U \cap g^{-1} H U \neq \varnothing$. Thus, $g f^{-1} \in H U U^{-1} H$. Since $U$ is an arbitrary neighborhood and $\left\{U U^{-1} \mid U \in \eta_{e}\right\}$ is a base of $\eta_{e}$, it follows that for all $U \in \eta_{e}, g f^{-1} \in H U H$, and (2) is shown.

Since all steps in the above proof are reversible, it follows that (2) implies (1). (2) and (3) are clearly equivalent. The result follows.

In view of (2.3), let $K(H)=\bigcap_{U \in \eta_{c}} H U H$. We now establish the connections between properties of $K(H)$ and $P(H \backslash G)$.

TheOREM (2.4). Consider the coset transformation group $\left(H^{*} \backslash G, G\right)$. Then the following statements are pairwise equivalent:

(1) $\left(H^{*} \backslash G, G\right)$ is a distal transformation group. 
(2) $K(H)=H^{*}$.

(3) For all $g \notin H^{*}$, there exists $U \in \eta_{e}$ such that $H U \cap g H=\varnothing$.

(4) For all $\mathrm{g} \notin H^{*}, e \notin \mathrm{cl}(\mathrm{HgH})$.

Proof. It is clear that (2) and (3), and (3) and (4) are equivalent. We show that (2) implies (1). Suppose $K(H)=H^{*}$. It is easy to see that $K\left(H^{*}\right)=\bigcap_{U \in n_{e}} H^{*} U H^{*}$ $=\bigcap_{U \in n_{e}} H U H=K(H)$, whence $K\left(H^{*}\right)=H^{*}$. Let $\left(H^{*} f, H^{*} g\right) \in P\left(H^{*} \mid G\right)$. Since $H^{*}$ is syndetic, (2.3) yields that $g f^{-1} \in K\left(H^{*}\right)=H^{*}$. Thus, $H^{*} g=H^{*} f$. It follows that $\left(H^{*} \backslash G, G\right)$ is distal.

We show that (1) implies (2). Let $g \in K\left(H^{*}\right)$. Since $g=g e^{-1},\left(H^{*}, H^{*} g\right) \in P\left(H^{*} \mid G\right)$. Thus, $H^{*}=H^{*} g$ and $g \in H^{*}$. Since $H^{*} \subset K\left(H^{*}\right)$, it follows that $H^{*}=K\left(H^{*}\right)=K(H)$. The proof is completed.

Since every distal transformation group is Hausdorff, we use $H^{*}$ instead of $H$ in (2.4).

THEOREM (2.5). The following statements are equivalent:

(1) $P(H \backslash G)$ is an equivalence relation.

(2) $K(H)$ is a subgroup of $G$.

Proof. We show that (1) implies (2). Let $f, g \in K(H)$. It is sufficient to show that $g f^{-1} \in K(H)$. Since $f=f e^{-1}, g=g e^{-1}$, it follows by (2.3) and the symmetry of $P(H \backslash G)$ that $(H f, H e),(H e, H g) \in P(H \backslash G)$. Thus, $(H f, H g) \in P(H \backslash G)$ and (2.3) yields that $g f^{-1} \in K(H)$.

We show that (2) implies (1). Let $(H f, H d),(H d, H g) \in P(H \backslash G)$. Then $d f^{-1}$, $g d^{-1} \in K(H)$. Thus, $g f^{-1}=\left(g d^{-1}\right)\left(d f^{-1}\right) \in K(H)$, and $(H f, H g) \in P(H \backslash G)$. It follows that $P(H \backslash G)$ is an equivalence relation. The proof is completed.

Since $K(H)$ is invariant under inversion, we need only show that $K(H)$ is a semigroup to show that it is a subgroup.

THEOREM (2.6). Consider the following statements:

(A) $P(H \backslash G)$ is closed.

(B) $K(H)$ is closed.

(C) $K(H)$ is a closed subgroup of $G$.

Then (A) is equivalent to (B) and (C) implies (B). If $H$ is closed, then (A), (B), and (C) are pairwise equivalent.

Proof. We show that (A) implies (B). Let $\left(g_{n}\right)$ be a net in $K(H)$ such that $g_{n} \rightarrow g$. Since for all $n, g_{n}=g_{n} e^{-1} \in K(H),\left(H e, H g_{n}\right) \in P(H \backslash G)$. Since $\left(H e, H g_{n}\right) \rightarrow(H e, H g)$ and $P(H \backslash G)$ is closed, then $(H e, H g) \in P(H \backslash G)$ and $g=g e^{-1} \in K(H)$. Thus, $K(H)$ is closed.

We show that (B) implies (A). Let $\left(\left(H f_{n}, H g_{n}\right)\right)$ be a net in $P(H \backslash G)$ such that $\left(H f_{n}, H g_{n}\right) \rightarrow(H f, H g)$. Then $H f_{n} \rightarrow H f$ and $H g_{n} \rightarrow H g$. We direct $\eta_{e}$ by $U \leqq V$ if $V \subset U$. Let $U \in \eta_{e}$. There exists $n(U)$ such that $H f_{n(U)} \in H U f$ and $H g_{n(U)} \in H U g$. Thus, $H f_{n(U)} \cap U f \neq \varnothing, H g_{n(U)} \cap H g \neq \varnothing$. Choose $h_{n(U)}, k_{n(U)} \in H$ such that

$$
h_{n(U)} f_{n(U)} \in U f \text { and } k_{n(U)} g_{n(U)} \in U g \text {. }
$$


It follows that the nets $\left(h_{n(U)} f_{n(U)}\right)$ and $\left(k_{n(U)} g_{n(U)}\right)$ converge to $f$ and $g$ respectively, whence $\left(k_{n(U)} g_{n(U)}\right)\left(h_{n(U)} f_{n(U)}\right)^{-1}=k_{n(U)} g_{n(U)} f_{n(U)}^{-1} h_{n(U)}^{-1} \rightarrow g f^{-1}$.

Let $U \in \eta_{e}$. Since $\left(H f_{n(U)}, H g_{n(U)}\right) \in P(H \backslash G)$, then $g_{n(U)} f_{n(U)}^{-1} \in K(H)$ and

$$
k_{n(U)} g_{n(U)} f_{n(U)}^{-1} h_{n(U)}^{-1} \in k_{n(U)} K(H) h_{n(U)}^{-1}=K(H) .
$$

Since $K(H)$ is closed, $g f^{-1} \in K(H)$ and $(H f, H g) \in P(H \backslash G)$. Thus, $P(H \backslash G)$ is closed.

That (C) implies (B) is obvious. Suppose that $H$ is closed. We show that (B) implies (C). Since $K(H)$ is closed, then $P(H \backslash G)$ is closed. Since $H \backslash G$ is Hausdorff, it follows by [1, Corollary 1] that $P(H \backslash G)$ is an equivalence relation. Then $K(H)$ is a group by (2.5). The proof is completed.

Let $g \in G$. Consider the proximal cell $(H g) P(H \mid G)=\{H f \mid(H g, H f) \in P(H \mid G)\}$. It is an immediate consequence of (2.4) that $H f \in(H g) P(H \backslash G)$ iff $f \in K(H) g$. We now show that properties of a single proximal cell reflect in the proximal relation.

Theorem (2.7). Suppose that there exists $g \in G$ such that $(H g) P(H \backslash G)$ is closed. Then $P(H \backslash G)$ is closed.

Proof. Choose $g \in G$ such that $(H g) P(H \backslash G)$ is closed. Let $\left(g_{n}\right)$ be a net in $K(H) g$ such that $g_{n} \rightarrow f$. Then $\left(H g_{n}\right)$ is a net in $(H g) P(H \backslash G)$ such that $H g_{n} \rightarrow H f$. Since $(H g) P(H \backslash G)$ is closed, $H f \in(H g) P(H \backslash G)$ and $f \in K(H) g$. It follows that $K(H) g$ is closed, whence $K(H)$ is closed. By (2.6), $P(H \backslash G)$ is closed. The result follows.

If $H$ is closed and $P(H \backslash G)$ is closed, then $P(H \backslash G)$ is also an equivalence relation (see (2.6)). We thus have:

Corollary (2.8). Let $H$ be closed. Then the following statements are pairwise equivalent:

(1) $P(H \backslash G)$ is closed.

(2) For some $g \in G,(H g) P(H \backslash G)$ is closed.

(3) For all $g \in G,(H g) P(H \backslash G)$ is closed.

THEOREM (2.9). The following statements are equivalent:

(1) $\left(H^{*} \mid G, G\right)$ is distal.

(2) Some point $H^{*}$ d is distal (i.e., $\left.\left(H^{*} d\right) P\left(H^{*} \mid G\right)=\left\{H^{*} d\right\}\right)$.

Proof. Clearly (1) implies (2). We show that (2) implies (1). Choose a distal point $H^{*} d$. Let $\left(H^{*} f, H^{*} g\right) \in P\left(H^{*} \mid G\right)$. Then $g f^{-1} \in K\left(H^{*}\right)$ and $g f^{-1} d \in K\left(H^{*}\right) d$. Thus, $H^{*} g f^{-1} d \in\left(H^{*} d\right) P\left(H^{*} \mid G\right)$, whence $H^{*} g f^{-1} d=H^{*} d$. It follows that $g f^{-1} \in H^{*}$ and $H^{*} f=H^{*} g$. The result follows.

The conclusions of (2.7)-(2.9) do not hold in general for minimal sets.

We give an example of a nondistal coset transformation group. Consider the nonabelian noncompact Lie group $G=G L(2, R)$, the general linear group. Consider the closed nonnormal subgroup

$$
H=\left\{\left[\begin{array}{ll}
x & y \\
0 & z
\end{array}\right] \mid x z \neq 0\right\} .
$$


It can be shown that $H$ is syndetic; in fact, if

$$
K=\left\{\left[\begin{array}{ll}
a & b \\
c & d
\end{array}\right]|| a|,| b|,| c|,| d \mid \leqq 1 \text { and } a d-b c=1\right\}
$$

then $K$ is compact and $G=H K$.

To show that $(H \backslash G, G)$ is not distal, it is sufficient by (2.4) to show that $e \in \operatorname{cl}(H g H)$, for some $g \notin H$. Let

$$
g=\left[\begin{array}{ll}
1 & 0 \\
1 & 1
\end{array}\right]
$$

Consider the following sequences in $H$ :

Then for all $n$,

$$
\left(g_{n}\right)=\left(\left[\begin{array}{cc}
n^{1 / n} & 0 \\
0 & 1 / n
\end{array}\right]\right) ; \quad\left(h_{n}\right)=\left(\left[\begin{array}{cc}
1 & 0 \\
0 & n^{1+1 / n}
\end{array}\right]\right), \quad n \geqq 1 .
$$

$$
g_{n} g h_{n}=\left[\begin{array}{cc}
n^{1 / n} & 0 \\
1 / n & n^{1 / n}
\end{array}\right],
$$

whence $g_{n} g h_{n} \rightarrow e$. Thus, $e \in \mathrm{cl}(\mathrm{HgH})$. Using (2.3), we have actually identified a nontrivial proximal pair, namely $(\mathrm{He}, \mathrm{Hg})$.

A similar analysis shows that if $G=S L(2, R)$, the special linear group and $H_{0}=H \cap S L(2, R)$, then $\left(H_{0} \mid G, G\right)$ is not distal.

3. Some general criteria for distal. We first introduce conditions whereby if $f:(X, T) \stackrel{\sim}{\rightarrow}(Y, T)$ denotes a transformation group homomorphism ( $f$ is continuous onto and for all $x \in X, t \in T, x t f=x f t)$ and $(Y, T)$ is distal, then $(X, T)$ is distal. The following lemma is used in this direction.

Lemma (3.1). Let $(X, T)$ be a transformation group, with $X$ a compact uniform space. Let $P(X)$ be the proximal relation of $X$. Let $S$ be a syndetic subgroup of $T$ and $Y$ be a S-invariant subset of $X$. Suppose that $(Y, S)$ is distal. Then if $y, w \in Y$ and $y \neq w,(y, w) \notin P(X)$.

Proof. Let $y, w \in Y$ such that $y \neq w$. Let $\alpha$ be an index of $X$ such that $(y, w) S$ $\cap(\alpha \cap(Y \times Y))=\varnothing$. Choose a compact subset $K$ of $T$ such that $T=S K$. Since $X$ is compact, there is an index $\beta$ of $X$ such that $\beta K^{-1} \subset \alpha[6$, Lemma 1.21]. It is direct to verify that $(y, w) T \cap \beta=\varnothing$. The result follows.

Suppose we have $f:(X, T) \stackrel{\sim}{\rightarrow}(Y, T)$. If $y \in Y$ and $P(y)$ denotes the period of $T$ at $y$, then $y f^{-1} P(y)=y f^{-1}$, whence $\left(y f^{-1}, P(y)\right)$ is a transformation group.

Combining these remarks, we now can show the desired result.

TheOREM (3.2). Let $(X, T)$ and $(Y, T)$ be transformation groups with $X$ and $Y$ compact uniform spaces. Let $f:(X, T) \stackrel{\sim}{\rightarrow}(Y, T)$. Suppose that $(Y, T)$ is distal and pointwise periodic. Suppose further that for all $y \in Y,\left(y f^{-1}, P(y)\right)$ is distal. Then $(X, T)$ is distal.

Proof. $P(X)$ and $P(Y)$ will denote the proximal relations of $X$ and $Y$ respectively. Let $x, z \in X$ such that $x \neq z$. Suppose $x f \neq z f$. If $(x, z) \in P(X)$, then $(x f, z f) \in P(Y)$, whence $x f=z f$. So $(x, z) \notin P(X)$. Suppose $x f=z f=y$. Then $x, z \in y f^{-1}$. Since $P(y)$ is syndetic and $\left(y f^{-1}, P(y)\right)$ is distal, then $(x, z) \notin P(X)$ by (3.1). The result follows. 
It is easy to see that if $(X, T)$ and $(Y, S)$ are transformation groups with $X$ and $Y$ uniform spaces, if $f: X \rightarrow Y$ is a unimorphism onto, if $g: T \rightarrow S$ is surjective, if for all $x \in X$ and $t \in T,(x t) f=(x f)(t g)$, and if $(X, T)$ is distal, then $(Y, S)$ is distal. For if $\hat{f}: X \times X \rightarrow Y \times Y$ is induced by $f$ and if $x, z \in X, \alpha \in \mathscr{U}(X)$ such that $(x, z) T \cap \alpha=\varnothing$, then $\alpha \hat{f} \in \mathscr{U}(Y)$ and

$$
\varnothing=[(x, z) T \cap \alpha] \hat{f}=(x, z) T \hat{f} \cap(\alpha \hat{f})=(x, z) \hat{f}(T g) \cap(\alpha \hat{f})=(x f, z f) S \cap(\alpha \hat{f}) .
$$

Using this remark, we can specialize (3.2) to coset transformation groups.

Corollary (3.3). Let $K$ be a subgroup of $G$ such that $K \subset H$. Suppose that $H$ is closed and $K^{*}$ is syndetic. Suppose further that $(H \backslash G, G)$ and the coset transformation group $\left(K^{*} \backslash H, H\right)$ are distal. Then the coset transformation group $\left(K^{*} \mid G, G\right)$ is distal.

Proof. Since $H$ is closed, $K^{*} \subset H$. Thus, we have the canonical map $f: K^{*} \backslash G$ $\rightarrow H \backslash G$. It is clear that $f:\left(K^{*} \mid G, G\right) \stackrel{\sim}{\rightarrow}(H \backslash G, G)$.

Now consider $H g \in H \backslash G$. If $P(H g)$ denotes the period of $G$ at $H g$, it is easily seen that $P(H g)=g^{-1} H g$, whence $P(H g)$ is syndetic. Thus, $(H \backslash G, G)$ is pointwise periodic.

Let $g \in G$. Then $H g f^{-1}=K^{*} \mid H g$ and $\left(H g f^{-1}, P(H g)\right)=\left(K^{*} \mid H g, g^{-1} H g\right)$. Let $\phi$ be the $g$-transition of $\left(K^{*} \mid G, G\right)$ and $\Psi: H \rightarrow g^{-1} H g$ the inner automorphism induced by $g$. Then $\phi: K^{*} \backslash H \rightarrow K^{*} \backslash H g$ is a unimorphism onto. Let $h, k \in H$. $\left(K^{*} h k\right) \phi=K^{*} h k g=K^{*} h g g^{-1} k g=\left(K^{*} h \phi\right)(k \Psi)$. Since $\left(K^{*} \backslash H, H\right)$ is distal, so is $\left(K^{*} \backslash H g, g^{-1} H g\right.$ ) by the above remark. Since $H \backslash G$ and $K^{*} \backslash G$ are compact Hausdorff spaces, it follows by (3.2) that $\left(K^{*} \mid G, G\right)$ is distal. The proof is completed.

We will show (see (3.9)) that $K^{*}$ is syndetic in $H$. Thus, $K^{*} \backslash H$ has a unique uniformity and no ambiguity arises in (3.3).

As remarked before, every distal transformation group has a Hausdorff phase space. Since we are interested in transformation groups with non-Hausdorff phase spaces, we consider a weaker variant of the notion of distal.

Definition (3.4). Let $(X, T)$ be a transformation group with a uniform phase space $X$ and uniformity $\mathscr{U}(X)$. Then $(X, T)$ is a weakly distal transformation group (with respect to $\mathscr{U}(X))$ if whenever $x, y \in X$ and $\mathrm{cl}(x) \neq \mathrm{cl}(y)$, then $x$ and $y$ are distal (with respect to $\mathscr{U}(X)$ ).

It is easy to see that if $P(X)$ is the proximal relation of $(X, T)$ and

$$
\theta(X)=\{(x, y) \mid \operatorname{cl}(x)=\operatorname{cl}(y)\}
$$

is the associated relation of $X$, then $(X, T)$ is weakly distal iff $P(X) \subset \theta(X)$. It follows that when $X$ is $T_{0}$, the notion of weakly distal and distal are equivalent. We now consider the notion of weakly distal when $X$ is not $T_{0}$.

Lemma (3.5). Let $(X, T)$ be a transformation group with a uniform phase space $X$ and uniformity $\mathscr{U}(X)$. Let $X_{0}$ be the associated space of $X$, i.e., the partition space induced by $\theta(X)$. Let $\Pi_{0}: X \rightarrow X_{0}$ be the canonical projection. Then:

(1) $\mathscr{U}(X)$ induces a compatible uniformity $\mathscr{U}\left(X_{0}\right)$ of $X_{0}$.

(2) $\mathrm{II}_{0}$ is uniformly continuous and uniformly open with respect to $\mathscr{U l}\left(X_{0}\right)$.

(3) $\left(X_{0}, T\right)$ is a transformation group with action $\left(x \Pi_{0}, t\right) \rightarrow x t \Pi_{0}$.

(4) $\Pi_{0}:(X, T) \stackrel{\sim}{\rightarrow}\left(X_{0}, T\right)$. 
Proof. If $\Pi_{0}^{*}: X \times X \rightarrow X_{0} \times X_{0}$ is induced by $\Pi_{0}$, then (1) and (2) follow from the fact that $\mathscr{U}\left(X_{0}\right)=\left\{\beta \mid \alpha \Pi_{0}^{*} \subset \beta\right.$, for some $\left.\alpha \in \mathscr{U}(X)\right\}$ is a compatible uniformity of $X_{0}$. Since $X_{0}$ is a star-open partition and for all $x \in X$ and $t \in T, x \Pi_{0} \Pi_{0}^{-1} t$ $=x t \Pi_{0} \Pi_{0}^{-1}$, then (3) follows by [6, Definition 1.39] and (4) is immediate.

THEOREM (3.6). Under the hypotheses of (3.5), the following statements are equivalent:

(1) $(X, T)$ is weakly distal (with respect to $\mathscr{U}(X))$.

(2) $\left(X_{0}, T\right)$ is distal (with respect to $\left.\mathscr{U}\left(X_{0}\right)\right)$.

Proof. We show that (1) implies (2). Let $x, y \in X$ such that $x \Pi_{0} \neq y \Pi_{0}$. Since $(X, T)$ is weakly distal, there is a closed index $\alpha$ of $X$ such that $(x, y) T \cap \alpha=\varnothing$. $\alpha \Pi_{0}^{*} \in \mathscr{U}\left(X_{0}\right)$. Suppose that there exists $t \in T$ such that $\left(x \Pi_{0}, y \Pi_{0}\right) t \in \alpha \Pi_{0}^{*}$. By (3.5), $\left(x \Pi_{0}, y \Pi_{0}\right) t=\left(x t \Pi_{0}, y t \Pi_{0}\right)$. Pick $(w, z) \in \alpha$ such that $\left(w \Pi_{0}, z \Pi_{0}\right)=\left(x t \Pi_{0}, y t \Pi_{0}\right)$. Then $\operatorname{cl}(w)=\operatorname{cl}(x t)$ and $\operatorname{cl}(z)=\operatorname{cl}(y t)$. Thus, $(x t, y t) \subset \operatorname{cl}(w) \times \operatorname{cl}(z)=\operatorname{cl}((w, z)) \subset \alpha$, which is a contradiction. It follows that $\left(x \Pi_{0}, y \Pi_{0}\right) T \cap \alpha \Pi_{0}^{*}=\varnothing$, and $\left(X_{0}, T\right)$ is distal.

We show that (2) implies (1). Let $x, y \in X$ such that $\operatorname{cl}(x) \neq \operatorname{cl}(y)$. Then $x \Pi_{0} \neq y \Pi_{0}$. If $\alpha_{0} \in \mathscr{U}\left(X_{0}\right)$ such that $\left(x \Pi_{0}, y \Pi_{0}\right) T \cap \alpha_{0}=\varnothing$ and if $\alpha \in \mathscr{U}(X)$ such that $\alpha \Pi_{0}^{*} \subset \alpha_{0}$, then it follows that $(x, y) T \cap \alpha=\varnothing$. Thus, $(X, T)$ is weakly distal. The proof is completed.

It is not difficult to see that $\phi_{0}:(H \backslash G)_{0} \rightarrow H^{*} \mid G$, induced by the canonical map $\phi: H \backslash G \rightarrow H^{*} \mid G$ and defined by $\left(H g \Pi_{0}\right) \phi_{0}=H^{*} g$, is a unimorphism onto and induces a transformation group isomorphism between $\left((H \backslash G)_{0}, G\right)$ and $\left(H^{*} \backslash G, G\right)$. It then follows by (3.6) that to study weakly distal actions in coset transformation groups, one need only study distal actions.

COROLLARY (3.7). The following statements are equivalent:

(1) $(H \backslash G, G)$ is weakly distal.

(2) $\left(H^{*} \mid G, G\right)$ is distal.

We now proceed to show that coset transformation group of nilpotent groups are distal. Some preliminary results are needed.

Lemma (3.8). Let $L$ and $M$ be subgroups of $G$ such that $L \triangleleft M$. Then $L^{*} \triangleleft M^{*}$.

Proof. If $g \in G$, let $\theta(g)$ be the topological inner automorphism of $G$ induced by $g$. Let $g \in M$. Since $L \theta(g)=L,\left(L^{*}\right) \theta(g)=(L \theta(g))^{*}=L^{*}$. Let $\left(g_{n}\right)$ be a net in $M$ such that $g_{n} \rightarrow g$. If $h \in L^{*}, g_{n}^{-1} h g_{n} \rightarrow g^{-1} h g$. Since for each $n, g_{n}^{-1} h g_{n} \in L^{*}$, so is $g^{-1} h g$. Thus, for all $g \in M^{*}, L^{*} \theta(g) \subset L^{*}$. The result follows.

Lemma (3.9). Let $L$ be a closed subgroup of $G$. Let $A \subset L$ such that $A$ is syndetic in $G$. Then $A$ is syndetic in $L$.

Proof. Let $K$ be a compact subset of $G$ such that $G=A K$. Let $C=K \cap L$. Since $L$ is closed, $C$ is compact. It is direct to verify that $L=A C$.

We will utilize the notion of a subgroup $L$ being subnormal in $G$ and denote it by $L \triangleleft \triangleleft G$. This means, of course, that there is a finite sequence $\left(L_{0}, L_{1}, \ldots, L_{n}\right)$ 
of subgroups of $G$ such that $L=L_{0} \triangleleft L_{1} \triangleleft \cdots \triangleleft L_{n}=G$. Such a sequence will be called a subnormal sequence for $L$. We finally note that, as an immediate consequence of (2.4), if $L$ is a closed syndetic normal subgroup of $G$, then $(L \mid G, G)$ is distal.

We now give the main result of $\S 2$.

THEOREM (3.10). Let $H \triangleleft \triangleleft G$. Then:

(1) $(H \backslash G, G)$ is weakly distal.

(2) $\left(H^{*} \mid G, G\right)$ is distal.

Proof. By (3.7), it is sufficient to show (2). Let $\left(A_{0}, A_{1}, \ldots, A_{n}\right)$ be a subnormal sequence for $H$. By $(3.8),\left(A_{0}^{*}, A_{1}^{*}, \ldots, A_{n}^{*}\right)$ is a subnormal sequence for $H^{*} . A_{i}^{*}$ is syndetic in $G(0 \leqq i \leqq n)$, since $H \subset A_{i}^{*}$. It follows by (3.9) that $A_{i}^{*}$ is a closed syndetic normal subgroup of $A_{i+1}^{*}(0 \leqq i \leqq n-1)$. Thus, the coset transformation group $\left(A_{i}^{*} \backslash A_{i+1}^{*}, A_{i+1}^{*}\right)$ is distal $(0 \leqq i \leqq n-1)$.

We complete the proof by showing inductively that $\left(A_{n-j}^{*} \backslash G, G\right)$ is distal $(1 \leqq j \leqq n)$. For $j=1,\left(A_{n-1}^{*} \mid G, G\right)=\left(A_{n-1}^{*} \mid A_{n}^{*}, A_{n}^{*}\right)$ is distal. Suppose $\left(A_{n-j}^{*} \mid G, G\right)$ is distal. Since $\left(A_{n-(j+1)}^{*} \mid A_{n-j}^{*}, A_{n-j}^{*}\right)$ is distal, $\left(A_{n-(j+1)}^{*} \mid G, G\right)$ is distal by (3.3). It follows that $\left(A_{0}^{*} \backslash G, G\right)=\left(H^{*} \backslash G, G\right)$ is distal. The proof is completed.

It is known that (see [12, Theorem (6.4.10)]) that every subgroup of a nilpotent group is subnormal. Thus, we immediately obtain from (3.10):

COROLlaRY (3.11). Let $G$ be a nilpotent group. Then:

(1) $(H \backslash G, G)$ is weakly distal.

(2) $\left(H^{*} \mid G, G\right)$ is distal.

In this direction see $[9, \S 2]$.

The problem of determining when a particular subgroup of a topological group is subnormal is quite difficult. For the blanket assumption of subnormality, it is a consequence of [13; see MR 27 5860] that if $G$ is a connected locally compact Hausdorff group, then $G$ being nilpotent is equivalent to every subgroup being subnormal.

We can generalize the notion of a subnormal sequence and still obtain results analogous to (3.10). In this direction, let $L$ be a subgroup of $G$. Let $\gamma$ be an ordinal number. Then an [increasing] [decreasing] subnormal chain for $L$ of order $\gamma$ is a family $\left(L_{\alpha} \mid \alpha \leqq \gamma\right)$ of subgroups of $G$ such that:

(1) $\left[L_{0}=L\right.$ and $\left.L_{\gamma}=G\right]\left[L_{0}=G\right.$ and $\left.L_{\gamma}=L\right]$.

(2) If $\beta \leqq \gamma$ and $\beta$ is a limit ordinal, then $\left[L_{\beta}=\bigcup_{\alpha<\beta} L_{\alpha}\right]\left[L_{\beta}=\bigcap_{\alpha<\beta} L_{\alpha}\right]$.

(3) If $\beta<\gamma$, then $\left[L_{\beta} \triangleleft L_{\beta+1}\right]\left[L_{\beta+1} \triangleleft L_{\beta}\right]$.

By using transfinition induction together with (3.3) and some additional results for the case of the limit ordinals, we have:

THEOREM (3.12). Suppose that for some ordinal $\gamma$, there exists a decreasing subnormal chain for $H$ of order $\gamma$. Then:

(1) $(H \backslash G, G)$ is weakly distal.

(2) $\left(H^{*} \backslash G, G\right)$ is distal. 
For the increasing case, we can show an analogous result when $\gamma=\omega$, the first infinite ordinal. The problem for larger ordinals is the analysis of the limit ordinal cases.

One can see that the converse of (3.12) does not hold even for solvable groups. Let $G=\operatorname{Sym}$ (3), the symmetric group on 3 symbols, treated as a discrete group. Let

$$
g=\left(\begin{array}{lll}
1 & 2 & 3 \\
2 & 1 & 3
\end{array}\right) \in G .
$$

Consider the subgroup $H=\{e, g\}$. Then trivally $H$ is closed and syndetic, and $(H \backslash G, G)$ is distal. It is easy to verify that the normalizer of $H$ in $G$ is $H$. It then follows that there is no increasing subnormal chain for $H$ of any order. Using the fact that $H$ is maximal in $G$ and nonnormal, one can show that there is no decreasing subnormal chain for $H$.

We now consider the problem of equicontinuity in coset transformation groups.

THEOREM (3.13). Let $G$ be a locally connected nilpotent group and let $H$ be discrete. Suppose that the left and right uniformities of $G$ do not coincide. Then $(H \backslash G, G)$ is weakly distal, point-to-point transitive, and nonequicontinuous. If $G$ is Hausdorff, we can replace weakly distal by distal.

Proof. It has been shown by Gottschalk, (see [5, p. 57]) that under the given hypotheses (without nilpotence), $(H \backslash G, G)$ is equicontinuous iff the left and right uniformities of $G$ coincide. Thus, $(H \backslash G, G)$ is point-to-point transitive and nonequicontinuous. By (3.11), $(H \backslash G, G)$ is weakly distal.

If $G$ is Hausdorff, then $H$ is closed, whence $(H \backslash G, G)$ is distal. The result follows.

As an example, consider the connected nilpotent Lie group

$$
G=\left\{\left[\begin{array}{lll}
1 & x & y \\
0 & 1 & z \\
0 & 0 & 1
\end{array}\right] \mid x, y, z \in \boldsymbol{R}\right\}
$$

and the discrete syndetic subgroup

$$
H=\left\{\left[\begin{array}{ccc}
1 & a & b \\
0 & 1 & c \\
0 & 0 & 1
\end{array}\right] \mid a, b, c \in \boldsymbol{Z}\right\}
$$

see [3, pp. 17, 35, 46-48]. To see that the left and right uniformities of $G$ do not coincide, consider the sequences

$$
\left(a_{n}\right)=\left(\left[\begin{array}{ccc}
1 & n & 1 / n \\
0 & 1 & 1 / n \\
0 & 0 & 1
\end{array}\right]\right) \text { and }\left(b_{n}\right)=\left(\left[\begin{array}{ccc}
1 & (1 / n)-n & 1 / n \\
0 & 1 & 1 / n^{2} \\
0 & 0 & 1
\end{array}\right]\right)
$$

Then $\lim _{n \rightarrow \infty} a_{n} b_{n}=e$ and $\lim _{n \rightarrow \infty} b_{n} a_{n} \neq e$. The result follows by [7, 4.24]. 
4. Applications to Lie groups. If $K$ and $L$ are subgroups of $G$, we will denote the commutator subgroup of $K$ and $L$ by $[K, L]$. Thus, $[K, L]$ is the subgroup generated by $\left\{g f g^{-1} f^{-1} \mid g \in K\right.$ and $\left.f \in L\right\}$.

Lemma (4.1). Let $G$ be Hausdorff and $K$ be a nilpotent subgroup of $G$. Then $K^{*}$ is nilpotent.

Proof. Let $\left(Z^{n}(K)\right)$ and $\left(G^{n}(K)\right)$ be the lower central series and topological lower central series for $K$ respectively. For some $m, Z^{m}(K)=\{e\}$. Consider the topological lower central series $\left(G^{n}\left(K^{*}\right)\right)$. It is an immediate induction to show that for all $n, G^{n}\left(K^{*}\right) \subset Z^{n}(K)^{*}$. Thus, $G^{m}\left(K^{*}\right) \subset Z^{m}(K)^{*}=\operatorname{cl}(e)=\{e\}$, and $G^{m}\left(K^{*}\right)=\{e\}$. The result follows.

Throughout the rest of $\S 4, G$ will denote a connected Lie group. By a subgroup of $G$, we will mean an analytic subgroup. Thus, $H$ will denote a syndetic subgroup. We let $R(G)$ denote the nil-radical of $G$, i.e., the connected normal nilpotent subgroup of $G$ which is maximal with respect to these properties. Since $G$ is connected, $R(G)$ is known to exist. It follows by $(4.1)$ that $R(G)$ is closed.

Since $R(G)$ is nilpotent, we can apply $\S 3$ to look for distal actions. The next result shows that in certain Lie groups $R(G)$ is sufficiently large and intersects certain syndetic subgroups syndetically.

THEOREM (4.2). Let $G$ be simply-connected and solvable. Suppose that $H$ does not contain any proper connected subgroup $L$ of $G$ such that $L \triangleleft G$. Then:

(1) $(H \cap R(G) \backslash R(G), R(G))$ is distal.

(2) $(H \backslash H R(G), R(G))$ is distal.

Proof. (1) $H$ is closed by [8, XII, Theorem 2.2]. Since $R(G)$ is closed, $R(G)$ is locally compact. $H \cap R(G) \backslash R(G)$ is compact by [11, $\S 11$, Prop. 1]. By [6, Remark 2.03], $H \cap R(G)$ is a closed syndetic subgroup of $G$. The result follows by (3.11).

(2) Consider the map $\phi: H \cap R(G) \backslash R(G) \rightarrow H \backslash H R(G)$ defined by $((H \cap R(G)) g) \phi$ $=H g$, for $g \in R(G)$. It is easily verified that $\phi$ is a continuous bijection. Since $H \cap R(G) \backslash R(G)$ is compact and $H \backslash H R(G)$ is Hausdorff, $\phi$ is a homeomorphism. Consider $\phi^{-1}$. Let $f, g \in R(G)$. Then $(H f g) \phi^{-1}=(H \cap R(G)) f g=(H f) \phi^{-1} g$. Thus. $\phi^{-1}$ is a transformation group isomorphism and the result follows.

When $H$ is discrete, we can get an analogous result without solvability.

THEOREM (4.3). Let $G$ be simply-connected with no compact normal subgroups other than $\{e\}$. Let $H$ be discrete. Then:

(1) $(H \cap R(G) \backslash R(G), R(G))$ is distal.

(2) $(H \backslash H R(G), R(G))$ is distal.

Proof. Since $H$ is closed and $H \cap R(G) \backslash R(G)$ is compact [2, Theorem 2], the proof proceeds exactly as in (4.2).

If $G$ is simply-connected and solvable, then $G$ satisfies the hypothesis of (4.3) [8, XII, Theorem 2.3]. It follows by either (4.2) or (4.3) that if $H$ is discrete, $(H \cap R(G) \backslash R(G), R(G))$ is distal. 
5. Point-transitive properties, and the existence of minimal distal subgroups. In this section we will be concerned with the action of a subgroup $K$ on $H \backslash G$. We investigate conditions which yield that $(H \backslash G, K)$ is either point-transitive or minimal distal. Finally, we remove the blanket assumption that $H$ is necessarily syndetic.

Let $T$ be a topological group and let $\theta: T \rightarrow G$ be a topological group homomorphism. Then the coset transformation group induced by $\theta$ is the transformation group $(H \backslash G, T, \pi(\theta))$ with action $(H g, t) \pi(\theta)=H g(t \theta)$. This notion generalizes our previous notion in $\S 1$, since id: $G \rightarrow G$ yields $(H \backslash G, G)$.

Lemma (5.1). Let $T$ be a topological group. Let $\theta: T \rightarrow G$ be a topological group homomorphism. Let $g \in G$. Then the following statements are pairwise equivalent:

(1) $\mathrm{Hg}$ is a transitive point of $(H \backslash G, T, \pi(\theta))$.

(2) $G=\operatorname{cl}(H g(T \theta))$.

(3) For every nonvacuous open subset $U$ of $G$, there exists $t \in T$ such that $g(t \theta) \in H U$.

Proof. The equivalence of (2) and (3) is clear. We note that $\mathrm{Hg}$ is a transitive point of $(H \backslash G, T, \pi(\theta))$ iff $H g$ is a transitive point of $(H \backslash G, T \theta)$. We show that (1) implies (2). Suppose $H g$ is a transitive point of $(H \backslash G, T, \pi(\theta))$. Then $\operatorname{cl}\{H g(t \theta) \mid t \in T\}$ $=H \backslash G$. Let $U$ be a nonvacuous open subset of $G$. There exists $t \in T$ such that $H g(t \theta) \subset H U$. Then $g(t \theta) \in H U$ and $H g(t \theta) \cap U \neq \varnothing$. It follows that $G=\operatorname{cl}(H g(T \theta))$. Since all the steps are reversible, the proof of (2) implies (1) follows in a similar fashion. The result follows.

In the following definition, we consider when there are sufficiently many topological group homomorphisms from $T$ to $G$.

Definition (5.2). Let $T$ be a topological group. Let $\operatorname{Hom}(T, G)$ be the set of all topological group homomorphisms from $T$ to $G$, provided with its point-open topology. Then $\operatorname{Hom}(T, G)$ [covers] [continuously covers] $G$ if there exists a [map][continuous map] $\theta: G \rightarrow \operatorname{Hom}(T, G)$, where $g \rightarrow \theta_{g}$, such that for all $g \in G$, $g \in T \theta_{g}$. Such a map $\theta$ is called a [covering][continuous covering] choice function.

We now show the main result of $\S 5$ (see [9, Theorem (3.1)]).

THEOREM (5.3). Let $T$ be a topological group. Let $G$ be a locally compact second countable group and let $f \in G$. Let $\theta: G \rightarrow \operatorname{Hom}(T, G)$, where $g \rightarrow \theta_{g}$, be a continuous covering choice function. Suppose that for every nonvacuous open subset $U$ of $G$, $G=\operatorname{cl}\left(H f\left(\bigcup_{g \in U} T \theta_{g}\right)\right)$. Then for almost all $g \in G$ (i.e., for $g$ in a comeager subset), $H f$ is a transitive point of $\left(H \backslash G, T, \pi\left(\theta_{g}\right)\right)$.

Proof. Let $\mathfrak{A}$ be a countable base for $G$ such that $\varnothing \notin \mathfrak{A}$. For all $A \in \mathfrak{A}$, let $E(A)=\left\{g \mid g \in G\right.$ and $\left.f\left(T \theta_{g}\right) \cap H A=\varnothing\right\}$. We show that $E(A)$ is closed. Let $\left(g_{n}\right)$ be a net in $E(A)$ such that $g_{n} \rightarrow g$. Now for all $n, f\left(T \theta_{g_{n}}\right) \subset G-(H A)$. Since $A$ is open, $G-(H A)$ is closed. Moreover, since $\theta$ is continuous, $\theta_{g_{n}} \rightarrow \theta_{g}$. Let $t \in T . f\left(t \theta_{g_{n}}\right)$ $\rightarrow f\left(t \theta_{g}\right)$, and $f\left(t \theta_{g}\right) \in G-(H A)$. It follows that $f\left(T \theta_{g}\right) \subset G-(H A)$ and $g \in E(A)$.

We show that int $E(A)=\varnothing$. Suppose int $E(A) \neq \varnothing$. Then $U=$ int $E(A)$ is a nonvacuous open set. Thus, $G=\operatorname{cl}\left(H f\left(\bigcup_{g \in U} T \theta_{g}\right)\right)$. Since $A \neq \varnothing, A \cap\left[H f\left(\bigcup_{g \in U} T \theta_{g}\right)\right] \neq \varnothing$. 
There exists $g \in U$ such that $A \cap H f\left(T \theta_{g}\right) \neq \varnothing$. Then $H A \cap f\left(T \theta_{g}\right) \neq \varnothing$, and $g \notin E(A)$. From this contradiction it follows that int $E(A)=\varnothing$.

Since $G$ is locally compact, $G$ is a Baire space. Since $E=\bigcup_{A \in \mathfrak{A}} E(A)$ is meager, $G-E$ is comeager and dense. Let $g \in G-E$. Let $V$ be a nonvacuous open subset of $G$. There exists $A \in \mathfrak{A}$ such that $A \subset V$. Since $f\left(T \theta_{g}\right) \cap H A \neq \varnothing$, then $f\left(T \theta_{g}\right) \cap H V \neq \varnothing$. By (5.1), $H f$ is a transitive point of $\left(H \backslash G, T, \pi\left(\theta_{g}\right)\right)$. The result follows.

Let $\phi: G \rightarrow G$ be a function. If we now assume that for every nonvacuous open subset $U$ of $G, G=\operatorname{cl}\left(H(U \phi)\left(\bigcup_{g \in U} T \theta_{g}\right)\right)$, and assume $\bigcap\{U \phi \mid U$ open in $G, U \neq \varnothing\}$ $\neq \varnothing$, then, by redefining $E(A)$ to be $\left\{g \mid g \in G\right.$ and $\left.(A \phi)\left(T \theta_{g}\right) \cap H A=\varnothing\right\}$, we can use the proof of (5.3) to show that for almost all $g,\left(H \backslash G, T, \pi\left(\theta_{g}\right)\right)$ is pointtransitive. The transitive points are $H f$, for $f \in \bigcap_{A \in \mathfrak{I}} A \phi$.

Let $g \in G$. We denote the cyclic subgroup generated by $g$ by $\mathscr{C}(g)$. We let $\phi_{g}: Z \rightarrow G$ be the topological group homomorphism defined by $n \phi_{g}=g^{n}$. If $A \subset G$, then $\mathscr{C}(A)=\bigcup_{g \in A} \mathscr{C}(g)$.

Since for all $n, g \rightarrow g^{n}$ from $G$ to $G$ is continuous, we note that $\phi: G \rightarrow \operatorname{Hom}(Z, G)$, where $g \rightarrow \phi_{g}$, is the canonical continuous covering choice function for $T=Z$. Using this fact, we can strengthen (5.3) when $T=Z$.

Corollary (5.4). Let $f \in G$. Consider the following properties:

(A) For every nonvacuous open subset $U$ of $G, G=\operatorname{cl}(H f \mathscr{C}(U))$.

(B) $\left\{g \mid H f\right.$ is a transitive point of $\left.\left(H \backslash G, Z, \pi\left(\phi_{g}\right)\right)\right\}$ is comeager in $G$.

If $G$ is locally compact, then (B) implies (A). If $G$ is locally compact second countable, then (A) and (B) are equivalent.

Proof. We show that (B) implies (A). Since $G$ is locally compact, $G$ is a Baire space. Let $D=\left\{g \mid H f\right.$ is a transitive point of $\left.\left(H \backslash G, Z, \pi\left(\phi_{g}\right)\right)\right\}$. Since $D$ is comeager, $D$ is dense. Let $U$ be a nonvacuous open subset of $G$ and $g \in D \cap U$. By (5.1), $G=\operatorname{cl}\left(H f\left(Z \phi_{g}\right)\right)=\operatorname{cl}(H f \mathscr{C}(g)) \subset \operatorname{cl}(H f \mathscr{C}(U))$, and $G=\operatorname{cl}(H f \mathscr{C}(U))$.

Now suppose $G$ is locally compact second countable. By using $\phi$ and noting that if $A \subset G, \mathscr{C}(A)=\bigcup_{g \in A} Z \phi_{g}$, it follows immediately by (5.3) that (A) implies (B). The proof is completed.

We now consider when $\left(H^{*} \backslash G, T, \pi(\theta)\right)$ is minimal distal. If $g \in G$, it is obvious that $\mathrm{cl}(H g(T \theta))=\mathrm{cl}\left(H^{*} g(T \theta)\right)$. It follows immediately by $(5.1)$ that $H g$ is a transitive point of $(H \backslash G, T, \pi(\theta))$ iff $H^{*} g$ is a transitive point of $\left(H^{*} \backslash G, T, \pi(\theta)\right)$. Using this, we obtain:

Lemma (5.5). Let $T$ be a topological group and $\theta \in \operatorname{Hom}(T, G)$. Let $H$ be syndetic in $G$ and let $(H \backslash G, G)$ be weakly distal. Suppose that $(H \backslash G, T, \pi(\theta))$ is point-transitive. Then $\left(H^{*} \mid G, T, \pi(\theta)\right)$ is minimal distal.

Proof. By (3.7), $\left(H^{*} \backslash G, G\right)$ is distal, whence $\left(H^{*} \backslash G, T, \pi(\theta)\right)$ is distal. It follows by [4, Theorem 1] that $\left(H^{*} \mid G, T, \pi(\theta)\right)$ is pointwise almost periodic. But since $(H \backslash G, T, \pi(\theta))$ is point-transitive, so is $\left(H^{*} \backslash G, T, \pi(\theta)\right)$. Since $H^{*} \backslash G$ is compact Hausdorff, it follows that $\left(H^{*} \mid G, T, \pi(\theta)\right)$ is minimal. The result follows.

The next result is an immediate consequence of (5.3) and (5.5). 
THEOREM (5.6). Let $H$ be syndetic in $G$ and let $(H \backslash G, G)$ be weakly distal. Suppose the hypotheses for (5.3) are satisfied for $G$, the topological group $T$, and some $f \in G$. Then for almost all $g \in G,\left(H^{*} \mid G, T, \pi\left(\theta_{g}\right)\right)$ is minimal distal.

When $T=Z$, we can again strengthen (5.6).

Corollary (5.7). Let $G$ be locally compact second countable. Let $H$ be syndetic in $G$ and let $(H \mid G, G)$ be weakly distal. Then the following statements are pairwise equivalent:

(1) There exists $f \in G$ such that for every nonvacuous open subset $U$ of $G$, $G=\operatorname{cl}(H f \mathscr{C}(U))$.

(2) There exists $f \in G$ such that $\left\{g \mid H f\right.$ is a transitive point of $\left.\left(H \backslash G, Z, \pi\left(\phi_{g}\right)\right)\right\}$ is comeager in $G$.

(3) $\left\{g \mid\left(H^{*} \mid G, Z, \pi\left(\phi_{g}\right)\right)\right.$ is minimal distal $\}$ is comeager in $G$.

Proof. The equivalence of (1) and (2) follows by (5.4). By (5.5), (2) implies (3). We show that (3) implies (2). Let $g \in G$ such that $\left(H^{*} \backslash G, Z, \pi\left(\phi_{g}\right)\right)$ is minimal distal. Then $H^{*} e$ is a transitive point of $\left(H^{*} \backslash G, Z, \pi\left(\phi_{g}\right)\right)$. Thus, $H e$ is a transitive point of $\left(H \backslash G, Z, \pi\left(\phi_{g}\right)\right)$. It follows that $\left\{g \mid H e\right.$ is a transitive point of $\left.\left(H \backslash G, Z, \pi\left(\phi_{g}\right)\right)\right\}$ is comeager in $G$. The result follows.

We finally consider the case when we simply have a covering choice function.

THEOREM (5.8). Let $G$ be locally compact second countable. Let $T$ be a topological group. Let $\theta: G \rightarrow \operatorname{Hom}(T, G)$, where $g \rightarrow \theta_{g}$, be a covering choice function. Let $f \in G$. Suppose that for every nonvacuous open subset $U$ of $G, G=\operatorname{cl}(H f \mathscr{C}(U))$. Then for almost all $g \in G, H f$ is a transitive point of both $\left(H \backslash G, Z, \pi\left(\theta_{g}\right)\right)$ and $\left(H \backslash G, T, \pi\left(\phi_{g}\right)\right)$. If, in addition, $H$ is syndetic in $G$ and $(H \backslash G, G)$ is weakly distal, then for almost all $g \in G,\left(H^{*} \mid G, Z, \pi\left(\phi_{g}\right)\right)$ and $\left(H^{*} \mid G, T, \pi\left(\theta_{g}\right)\right)$ are minimal distal.

Proof. The part of the first assertion about $Z$ follows from (5.4). Let $g \in G$ such that $H f$ is a transitive point of $\left(H \backslash G, Z, \pi\left(\phi_{g}\right)\right)$. By $(5.1), G=\operatorname{cl}(H f \mathscr{C}(g))$. Since $g \in T \theta_{g}$, then $\mathscr{C}(g) \subset T \theta_{g}$, whence $G=\operatorname{cl}\left(H f\left(T \theta_{g}\right)\right)$. Again by (5.1), Hf is a transitive point of $\left(H \backslash G, T, \pi\left(\theta_{g}\right)\right)$, and the first statement of the conclusion follows. The last part follows by (5.5).

If we replace the condition that $(H \backslash G, G)$ is weakly distal by $H \triangleleft \triangleleft G$, it follows by (3.10) that the conclusion of (5.8) still holds. Thus, we obtain [9, Theorem (3.2)].

Now let $G$ be a Lie group and $L(G)$ its Lie algebra. Suppose that exp: $L(G) \rightarrow G$ is onto. Let $g \in G$ and $X_{g} \in g \exp ^{-1}$. Let $\theta_{g}: R \rightarrow G$, defined by $t \theta_{g}=\exp t X_{g}$, be the induced 1-parameter subgroup. Then $\theta: G \rightarrow \operatorname{Hom}(\boldsymbol{R}, G)$ is a covering choice function.

Let $G$ be a connected simply-connected nilpotent Lie group. Then exp is a homeomorphism onto [10], and $G$ is clearly second countable. Using the covering choice function $\theta$ defined above, we obtain:

COROLlaRY (5.9). Let $G$ be a connected simply-connected nilpotent Lie group. Let $H$ be discrete syndetic. Then:

(1) For almost all $g \in G,\left(H \backslash G, Z, \pi\left(\phi_{g}\right)\right)$ is minimal distal.

(2) For almost all $g \in G,\left(H \backslash G, R, \pi\left(\theta_{g}\right)\right)$ is minimal distal. 
Proof. Clearly $H$ is closed. By (3.11), $(H \backslash G, G)$ is distal. By (5.7), it is sufficient to show that for every nonvacuous open subset $U$ of $G, G=\operatorname{cl}(H \mathscr{C}(U))$. But this is an immediate consequence of [3, Lemma, pp. 54-55]. The result follows.

We finally note that $[3, \mathrm{IV}$, Theorem 5 and Corollary] are consequences of (5.9).

\title{
REFERENCES
}

1. J. Auslander, On the proximal relation in topological dynamics, Proc. Amer. Math. Soc. 11 (1960), 890-895.

2. L. Auslander, On radicals of discrete subgroups of Lie groups, Amer. J. Math. 85 (1963), 145-150.

3. L. Auslander, L. Green, F. Hahn et al., Flows on homogeneous spaces, Annals of Mathematics Studies No. 53, Princeton Univ. Press, Princeton, N. J., 1963.

4. R. Ellis, Distal transformation groups, Pacific J. Math. 8 (1958), 401-405.

5. W. Gottschalk, A study of minimal sets, Ann. Inst. Fourier (Grenoble) 14 (1964), 55-60.

6. W. Gottschalk and G. Hedlund, Topological dynamics, Colloq. Publ., Vol. 36, Amer. Math. Soc., Providence, R. I., 1955.

7. E. Hewitt and K. Ross, Abstract harmonic analysis, Vol. 1, Springer-Verlag, New York, 1963.

8. G. Hochschild, The structure of Lie groups, Holden-Day, San Francisco, Calif., 1965.

9. H. Keynes, Topological dynamics in coset transformation groups, Bull. Amer. Math. Soc. 72 (1966), 1033-1035.

10. A. Malcev, On a class of homogeneous spaces, Amer. Math. Soc. Transl. 39 (1949).

11. G. D. Mostow, Factor spaces of solvable Lie groups, Ann. of Math. 60 (1954), 1-27.

12. W. R. Scott, Group theory, Prentice-Hall, Englewood Cliffs, N. J., 1964.

13. V. I. Ušakov, Topological groups with normalizer condition for closed subgroups, Izv. Akad. Nauk SSSR Ser. Mat. 27 (1963), 943-948. (Russian)

\author{
WeSLEYAN UNiversity, \\ Middletown, Connecticut \\ UNIVERSITY OF CALIFORNIA, \\ Santa Barbara, California
}

